\title{
Kink dynamics in spatially inhomogeneous media: The role of internal modes
}

\author{
Jorge A. González* \\ Centro de Física, IVIC, A.P. 21827, Caracas 1020-A, Venezuela \\ Sara Cuenda \\ Grupo Interdisciplinar de Sistemas Complejos (GISC), Departamento de Matemáticas, Universidad Carlos III de Madrid, \\ 28911 Leganés, Madrid, Spain \\ Angel Sánchez \\ Grupo Interdisciplinar de Sistemas Complejos (GISC), Departamento de Matemáticas, Universidad Carlos III de Madrid, \\ 28911 Leganés, Madrid, Spain \\ and Instituto de Biocomputación y Física de Sistemas Complejos (BIFI), Universidad de Zaragoza, Zaragoza, Spain
}

(Received 9 January 2007; published 23 March 2007)

\begin{abstract}
The phenomenon of length-scale competition in soliton-bearing equations perturbed by spatially dependent terms [A. Sánchez and A. R. Bishop, SIAM Rev. 40, 579 (1998)] is analyzed from a general viewpoint. We show that the perturbation gives rise to an effective potential for solitons, which consists of wells and barriers. We calculate the effect of these potential features on the solitons, establishing a direct relationship between the maxima, minima, and curvature of the potential with soliton deformations. When the typical wavelength of the perturbation is of the order of the soliton width, these deformations are seen to correspond to the excitation of shape modes and can lead to the dissipation of the soliton kinetic energy and, further, to the impossibility of soliton propagation. Thus, we demonstrate that the mechanism for length-scale competition is related to changes in the dynamics of the internal modes. We study different examples where the perturbation is introduced parametrically and nonparametrically to make it clear that our results apply to a wide class of equations.

DOI: 10.1103/PhysRevE.75.036611

PACS number(s): 05.45.Yv, 02.30.Hq, 02.30.Jr, 02.60.-x
\end{abstract}

\section{INTRODUCTION}

Soliton-bearing partial differential equations have been a very successful, widely applicable family of models for more than 40 years $[1,2]$. One of the most important of these models is the nonlinear Klein-Gordon equation, very relevant in many different contexts, ranging from particle physics [3] through biomolecules $[4,5]$ to superconducting devices $[6]$ or structural phase transitions [7]. On the other hand, the nonlinear Klein-Gordon equation is a paradigm of partial differential equations supporting topological soliton [8] solutions, this being the chief reason for such a general applicability. In this context, a very important issue is whether the topological soliton solutions of these model equations are robust when subjected to perturbations, which are unavoidable when modeling actual systems or problems [9].

Among the types of perturbations to be considered, a prominent case is that of disorder or spatial inhomogeneities, described by nonautonomous terms dependent on the spatial coordinates [10]. Such perturbations are ubiquitous in applications, as most real systems unavoidably deviate from the perfect homogeneous situation that corresponds to the unperturbed equation. Generally speaking, topological solitons are much more stable against perturbations $[8,10]$ than other types of excitations, mostly because the conservation of an associated quantity called topogical charge prevents their destruction. However, they are sensitive to perturbations of a typical length scale when this scale matches that of the natu-

\footnotetext{
*Electronic address: jorge@ivic.ve
}

ral width of the solitons, a phenomenon that has been termed length-scale competition [11-14]. Specifically, it was observed that topological solitons, also referred to as kinks, did not propagate in a spatially periodic potential of wavelength of the order of the kink width, whereas propagation was possible in potentials of smaller and larger wavelengths: In these two cases the soliton was simply unaffected by the potential or else behaved as a rigid particle moving on an effective periodic potential, respectively. In spite of the fact that several works have subsequently been devoted to understand this problem [15-26], we still lack a clear insight into it; indeed, most of the studies used only numerical simulations, and no simple analytical results were available.

The aim of the present paper is to contribute to this problem by means of an analytical study of the equation

$$
\phi_{t t}-\phi_{x x}+[1+\epsilon \cos (k x)] \frac{\partial U}{\partial \phi}=0,
$$

which we carry out with a collective coordinate technique [11] that allows for a drastic reduction of the number of degrees of freedom involved in the problem, while keeping a very good agreement with the numerical simulations [24]. In addition, we will also investigate kink stability in the related problem

$$
\phi_{t t}-\phi_{x x}+\frac{\partial U}{\partial \phi}=F(x),
$$

where $F(x)$ are suitably chosen functions such that the stability problem can be solved exactly. In carrying out this parallel study, we will find connections between both prob- 
lems that will provide a good basis to address the mechanisms underlying length-scale competition while, on the other hand, supporting the generality of our ideas and intepretation. Indeed, the main point we want to make in this paper is that length-scale competition is originated by the coupling between soliton deformations and perturbation features, such as the maxima, minima, and curvature of the corresponding effective potential. In order to present these results in detail, the paper is organized as follows: In Sec. II, we describe the collective coordinate approach to the periodically modulated nonlinear Klein-Gordon equation (1). By analyzing the equilibrium points of the corresponding effective potential, we will be able to find the region related to length-scale competition. We will then proceed with Eq. (2) in Sec. III, where we will consider three different choices for $F(x)$ in order to shed more light on this problem; specifically, we will show how solitons are affected by the perturbation in terms of excitation of shape modes. Finally, Sec. IV collects our results and their corresponding discussion.

\section{COLLECTIVE COORDINATE APPROACH FOR THE PERIODICALLY MODULATED EQUATION}

\section{A. Collective coordinate equations}

Collective coordinate techniques are a family of perturbative approximations to soliton-bearing partial differential equations whose main aim is to perform a reduction of degrees of freedom such that the problem is cast in the form of ordinary differential equations $[6,10,11]$. Typically, but not necessarily, this is accomplished by focusing on the effect of the perturbation on magnitudes characterizing the soliton solution of interest, such as the position of its center or its width.

The specific problem (1) was considered in [24]. That work is the only one to our knowledge where length-scale competition has been studied in terms of collective coordinates. The procedure begins by proposing the following ansatz:

$$
\phi(x, t)=\phi_{k}\{[x-X(t)] / l(t)\},
$$

where $\phi_{k}$ stands for the unperturbed one-soliton solution. The key idea of the technique is to derive equations for the two functions $X(t)$ and $l(t)$ that represent, respectively, the center and the width of the kink. Underlying this is the hypothesis that the effect of the perturbation is mainly to change these two quantities, without appreciably distorting the general shape of the kink solution. This, of course, must be checked a posteriori by numerical simulations of the full partial differential equation. The next step is now to use the Lagrangian approach developed in [25] to find the equations for the two collective coordinates. By introducing the ansatz (3) in the Lagrangian for Eq. (1), we find

$$
L=\frac{M_{0} l_{0}}{2 l} \dot{X}^{2}+\frac{\alpha M_{0} l_{0}}{2 l} \dot{l}^{2}-V(X, l),
$$

where

$$
V(X, l)=\frac{M_{0}}{2}\left(\frac{l_{0}}{l}+\frac{l}{l_{0}}\right)+\frac{\epsilon}{k} \cos (k X) W(k l) .
$$

The constants $M_{0}$ and $l_{0}$ represent the soliton "mass" and natural width, and their specific values depend on the function $U(\phi)$ considered. Particularly relevant instances of the nonlinear Klein-Gordon equation are the sine-Gordon equation, corresponding to $U(\phi)=1-\cos \phi$, and the $\phi^{4}$ equation, given by $U=\left(1-\phi^{2}\right)^{2} / 4$. The former is a fully integrable model in the absence of perturbations, with $\mathrm{N}$-soliton solutions for all $N$, whereas the latter is nonintegrable and possesses only one-soliton (or anti-soliton) solutions. It is then important to consider both examples in order to detect any difference between them arising from the rare property of exact integrability. For the sine-Gordon equation, $M_{0}=8$, $l_{0}=1$, and $\alpha=\pi^{2} / 12$, and the soliton solution is given by

$$
\phi(x, t)=4 \arctan \{\exp [x-X(t)] / l(t)\},
$$

leading to [24]

$$
W(y)=\frac{2 \pi y^{2}}{\sinh \left(\frac{\pi y}{2}\right)},
$$

in Eq. (5). On the other hand, for the $\phi^{4}$ equation, $M_{0}=4 / 3 \sqrt{2}, l_{0}=\sqrt{2}, \alpha=\left(\pi^{2}-6\right) / 12$, and

$$
W(y)=\frac{\pi y^{2}\left(y^{2}+4\right)}{24 \sinh \left(\frac{\pi y}{2}\right)} .
$$

Once we have the Lagrangian (4), we can immediately obtain the corresponding Euler-Lagrange equations for the two variables $X$ and $l$, finding

$$
\dot{P}=\epsilon \sin (k X) W(k l),
$$

$$
\dot{Q}=-\frac{1}{2 M_{0} l_{0}}\left(P^{2}+\frac{Q^{2}}{\alpha}\right)+\frac{M_{0} l_{0}}{2}\left(\frac{1}{l^{2}}-\frac{1}{l_{0}^{2}}\right)-\epsilon \cos (k X) W^{\prime}(k l),
$$

with

$$
P=\frac{M_{0} l_{0} \dot{X}}{l}, \quad Q=\frac{\alpha M_{0} l_{0} i}{l} .
$$

In [24], these equations were integrated numerically, and it was found that, in accordance with the numerical simulations of the full equation (1), there is a regime in which the dynamics of $P$ and $Q$ corresponds to a situation in which the motion of the kink in the full partial differential equation is stopped. For both the sine-Gordon and $\phi^{4}$ equations, this regime takes place for an interval of wavelengths centered around $\lambda=2 \pi / k=3$-i.e., for values of the perturbation wavelength close to the natural width of the kink. This agrees with the previous observations of length-scale competition [11,14], but being only numerical information, it did not provide any insight into the mechanisms responsible for such a phenomenon. We will now address this question by analyzing in more depth the collective coordinate equations. 


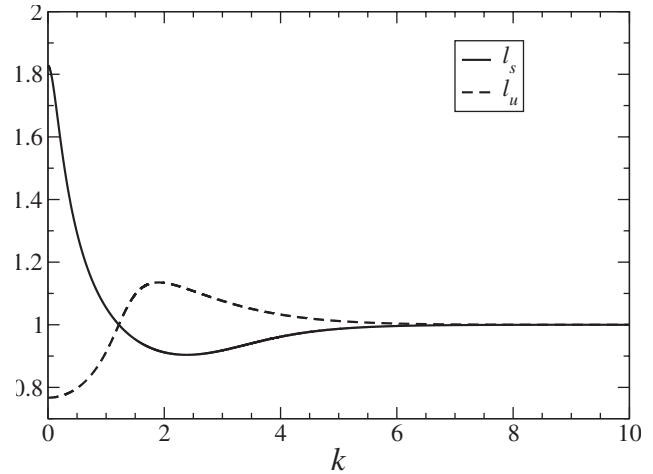

FIG. 1. Width values for stable and unstable equilibria as a function of $k$ for the collective coordinate approximation to the perturbed equation (1) with sine-Gordon potential. The perturbation parameter is $\epsilon=0.7$.

\section{B. Analysis of the collective coordinate equations}

We begin by looking for the equilibrium points of the system (9) and (10). We will carry out the calculation for the sine-Gordon case, but the procedure applies straightforwardly to the $\phi^{4}$ equation with equally good results. These are computed by imposing $\dot{X}=0$ and $\dot{l}=0$, which leads to

$$
\begin{gathered}
\epsilon \sin (k X) W(k l)=0 \\
\frac{M_{0} l_{0}}{2}\left(\frac{1}{l^{2}}-\frac{1}{l_{0}^{2}}\right)-\epsilon \cos (k X) W^{\prime}(k l)=0 .
\end{gathered}
$$

From these expressions we find that stable equilibria are given by

$$
\begin{gathered}
X_{s}=\frac{(2 n+1) \pi}{k}, \\
\frac{M_{0} l_{0}}{2}\left(\frac{1}{l_{s}^{2}}-\frac{1}{l_{0}^{2}}\right)=2 \pi \epsilon k l_{s}\left\{\frac{\frac{\pi}{2} k l_{s}-2 \tanh \left(\frac{\pi k l_{s}}{2}\right)}{\sinh \left(\frac{\pi k l_{s}}{2}\right) \tanh \left(\frac{\pi k l_{s}}{2}\right)}\right\},
\end{gathered}
$$

and unstable ones correspond to

$$
\begin{gathered}
X_{u}=\frac{2 n \pi}{k}, \\
\frac{M_{0} l_{0}}{2}\left(\frac{1}{l_{u}^{2}}-\frac{1}{l_{0}^{2}}\right)=-2 \pi \epsilon k l_{u}\left\{\frac{\frac{\pi}{2} k l_{u}-2 \tanh \left(\frac{\pi k l_{u}}{2}\right)}{\sinh \left(\frac{\pi k l_{u}}{2}\right) \tanh \left(\frac{\pi k l_{u}}{2}\right)}\right\} .
\end{gathered}
$$

From these expressions we can already extract some information about the equilibria. The values $l_{s}$ and $l_{u}$ obtained from them are plotted in Fig. 1, which has been obtained for $\epsilon=0.7$, as in the study of [24]. First, it is evident that in the limit $k \rightarrow \infty$-i.e., vanishing perturbation wavelength-the values for the width at equilibria go to the natural width $l_{0}$. Second, it can be seen that there is a critical value $k_{c}$ such that for $k=k_{c}, l_{u}=l_{s}=l_{0}$. Above that value, $k>k_{c}$, we find that $l_{u}>l_{0}$ and $l_{s}>l_{0}$, whereas in the other case, $k<k_{c}$, the situation is reversed, $l_{u}<l_{0}$ and $l_{s}<l_{0}$.

To go beyond these first results and to study the behavior of these equilibria as a function of $k$ in more detail, we can parametrize the values for $l_{s}$ and, correspondingly, $k_{s}$ in terms of a parameter $z$,

$$
\begin{gathered}
l_{s}(z)=\frac{l_{0}}{\sqrt{1-\frac{4 \pi \epsilon l_{0} z}{M_{0}}\left[\frac{2 \tanh \left(\frac{\pi z}{2}\right)-\frac{\pi}{2} z}{\left.\sinh \left(\frac{\pi z}{2}\right) \tanh \left(\frac{\pi z}{2}\right)\right]}\right.},} \\
k_{s}=\frac{z}{l_{s}(z)},
\end{gathered}
$$

and the same can be done for $l_{u}$ and $k_{u}$,

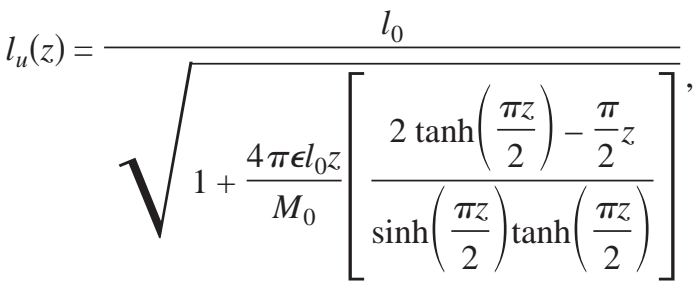

$$
\begin{aligned}
& k_{u}=\frac{z}{l_{u}(z)},
\end{aligned}
$$

where the parameter $z \in[0, \infty)$. With these expressions, one can analyze the dependence of the values of $l$ for the equilibria on $k$, finding that there is an extremum for certain $k_{m}$. This extremum turns out to be a maximum for $l_{u}$ and a minimum for $l_{s}$, as is depicted in Fig. 1 . This is a very important result indeed: the maximum for the unstable case is obtained for

$$
k_{m}=k_{u}\left(z_{m}\right)=z_{m} / l_{u}\left(z_{m}\right),
$$

with $z_{m} \approx 2(2+\sqrt{2}) / \pi$, which implies very good agreement with the simulation results for the range of wavelengths in which length-scale competition is observed: This value corresponds to $k_{m}=1.9$-i.e., $\lambda=3.3$-values for which the simulations in [24] showed the clearest evidence of lengthscale competition with the kink being trapped by the potential very rapidly.

Another important quantity is the second derivative of the effective potential $V(X, l)$ along the $X$ direction, evaluated at the equilibrium points. The absolute value of this derivative, $|C|$, yields a measure of the "curvature" of the potential on which the kink is moving and is given by

$$
|C|=\frac{2 \pi \epsilon l_{x}^{2} k^{3}}{\sinh \left(\frac{\pi l_{x} k}{2}\right)},
$$

where the subscript $x$ stands for $u$ or $s$, depending on the type of point one is looking at. The dependence of this magnitude 


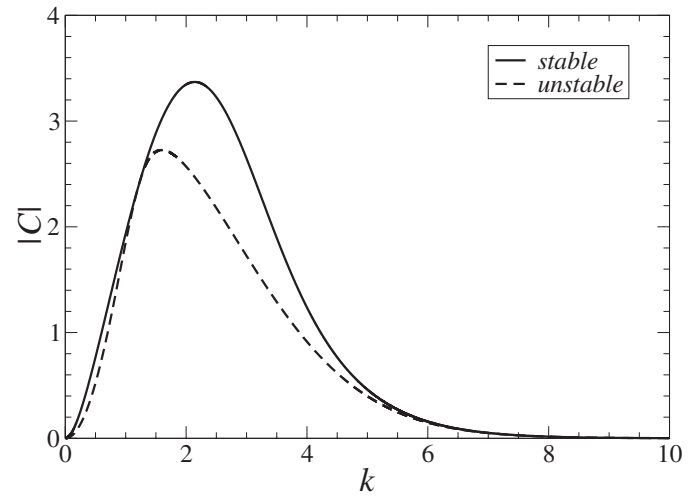

FIG. 2. Curvature of the potential at the stable and unstable equilibria as a function of $k$ for the collective coordinate approximation to the perturbed equation (1) with sine-Gordon potential. The perturbation parameter is $\epsilon=0.7$.

on $k$ is shown in Fig. 2. This plot is in agreement with the fact that one can easily show that when either $k \rightarrow \infty$ or $k \rightarrow 0,|C| \rightarrow 0$. On the other hand, the curvature has a maximum for a value of $k$ that is approximately given by $z_{m} \approx 6 / \pi$, corresponding to $k_{m}=1.6, \lambda=3.9$. This means that in the length-scale competition region of wavelengths, the kink is moving on a curved potential, whereas outside of this region, the effective potential is almost flat, for both small and large $k$. The relevance of this quantity for understanding length-scale competition will be made clear after the next section.

\section{SPATIALLY DEPENDENT FORCING TERMS}

After considering the case of the spatially periodic potential, we now turn our attention to Eq. (2), where the term $F(x)$ represents a constant, spatially dependent force acting on the system and can have one or more zeros. As we will see below, although this is a different problem, in which the perturbation enters additively, the phenomenology will allow us to shed light on the results of the preceding section.

Much as in the previous case, the system can be studied applying a collective coordinate technique $[6,18,26,27]$ to Eq. (2) with the ansatz (6); one can show that zeros of $F(x)$ represent equilibrium positions for the kink center of mass. Indeed, following the ideas in $[6,28]$, it can be shown that, restricting ourselves for simplicity to the case $i=0$ (i.e., one collective coordinate only), the effective force acting on the kink center is

$$
F_{e f f}(X) \propto \int_{-\infty}^{\infty} d x F(x) \frac{\partial \phi_{k}}{\partial x}\left(\frac{x-X}{l}\right),
$$

which for smooth choices of $F(x)$ leads to the above conclusion (see also [27] for an argument based on energetic considerations). On the other hand, the stability condition for the equilibria, $F\left(x_{*}\right)=0$, is given by

$$
\left.\frac{\partial F}{\partial x}\right|_{x_{*}}>0 .
$$

In the following, we will consider several examples for $F(x)$, for either the sine-Gordon or the $\phi^{4}$ equations, such that the exact kink solution of the perturbed equation can be obtained and the stability problem can be solved exactly. These examples show what one can expect of other topological soliton-bearing equations, where exact solutions are not available, such as that studied in Sec. II.

\section{A. Case I}

Let us consider the sine-Gordon equation, and let us choose

$$
F_{I}(x)=\frac{2\left(B^{2}-1\right) \sinh (B x)}{\cosh ^{2}(B x)},
$$

which has a unique zero at $x_{*}=0$. This point is an equilibrium point for the kink center of mass; for $B^{2}>1\left(B^{2}<1\right)$, the equilibrium is stable (unstable). The exact solution for the kink located at the equilibrium point is

$$
\phi(x)=4 \arctan [\exp (B x)] .
$$

Stability analysis leads to the following eigenvalue problem:

$$
\hat{L} f(x)=r f(x),
$$

where $\hat{L} \equiv-\partial_{x}^{2}+\left[1-2 \cosh ^{-2}(B x)\right]$ and $r=-\Lambda^{2}$, while $f(x)$ are the soliton modes describing small oscillations around the kink of the form $f(x) \exp (\Lambda t)$. Generally speaking, perturbation problems for soliton bearing equations should be studied by means of this operator technique [10], insofar as the eigenfunctions and eigenvalues allow one to compute the complete dynamics of the kink, since they form a complete basis for the space of solutions of the equation. However, in most cases this approach requires an exact shape of the perturbed kink and, in addition, one should be able to solve the eigenvalue problem, two steps that render the problem intractable. Therefore, the interest of the examples we discuss here is that for these specific choices, we can go through both stages of the process exactly, obtaining a great deal of information about the kink behavior. For the case under consideration here, we find that the eigenvalues of the discrete spectrum are given by $[17,26]$ (see also [29-31] for additional results and information on this type of problem)

$$
r_{n}=B^{2}\left(L+2 L n-n^{2}\right)-1,
$$

with $L(L+1)=2 / B^{2}$. The integer part of $L$ represents the number of eigenvalues in the discrete spectrum, corresponding to the soliton modes. This includes the translation (Goldstone) mode $r_{0}$ that arises from the breaking of the spatial invariance and (possibly) several internal modes $r_{n}$ with $n>0$ corresponding to the rest of the discrete spectrum. The continuum spectrum represents extended deformations of the soliton and can thus be identified with radiation.

Let us consider first the case $B^{2}<1$ (for $B=1$ the perturbation vanishes and we recover the spectrum of the unper- 
turbed sine-Gordon equation, with the discrete spectrum consisting only of the translation mode with zero eigenvalue). For these values of $B$, one can show, by means of a number of different methods, that the kink is in an equilibrium position of the potential, but its stability depends on the specific value of $B$. For $1 / 3<B^{2}<1$, the translational mode is unstable, meaning that the kink is placed on a "hill" and therefore that small perturbations can make the kink move away from equilibrium. In this situation there are no internal modes. The first one appears when $1 / 6<B^{2}<1 / 3$ : In this interval, there is an internal mode besides the translation one. For even lower values of $B^{2}$, further internal modes can be activated. When $B^{2}<2 /\left[L_{*}\left(L_{*}+1\right)\right]$, with $L_{*}=(5+\sqrt{17}) / 2$, the first internal mode becomes unstable. This instability in turn leads to destabilization of the whole kink solution, in the form of a "solitonic reaction" of the type 1 kink $\rightarrow 1$ antikink +2 kinks, which preserves the topological charge.

The connection of these results to the preceding section can be made clear by the following considerations: We can obtain information about the width by noting from Eq. (27) that the kink width is given $l=B^{-1}$. This means that when the kink is inside the potential well, its width is reduced. On the other hand, when the kink is on the "hill" of a potential barrier, its width is increased. We then see that as the parameter $B$ decreases, the kink width increases along with the number of activated internal modes, placing the kink in a situation closer to length-scale competition.

The case $B^{2}>1$ is also interesting, corresponding to the situation when the kink is inside a potential well. As we have just seen, the kink width is reduced, but in addition, a very surprising fact is found: namely, that the translational mode does not exist anymore, which suggests that the motion of the kink center inside the potential well is difficult; i.e., the kink is literally trapped in the potential well. For more details about this case we refer the reader to [26].

\section{B. Case II}

As our second example, we discuss now a more complicated situation. Let us consider the $\phi^{4}$ equation and

$$
F_{I I}(x)=\frac{1}{2} A \tanh (B x)\left[\epsilon_{1}+\epsilon_{2} \cosh ^{-2}(B x)\right] .
$$

Note that for $A=1$ and $B=1 / 2$, the perturbation vanishes and one recovers the usual, unperturbed $\phi^{4}$ equation. In this section, we use for convenience the $\phi^{4}$ potential written with an extra factor of $1 / 2$-i.e., $U=\left(1-\phi^{2}\right)^{2} / 8$. Depending on the values of the parameters, $F_{I I}$ can have either one or three zeros. In view of this, this force is a very suitable model for testing the pitchfork bifurcation phenomena in soliton dynamics, where changing the parameters we can have a situation with one equilibrium position transformed in another with three equilibria. Here, we will concentrate on the choice $\epsilon_{1}=A^{2}-1$ and $\epsilon_{2}=\left(4 B^{2}-A^{2}\right)$, for which the exact kink solution is very simple: $\phi(x)=A \tanh (B x)$ [18].

To analyze the stability, we again have an eigenvalue problem given by Eq. (28) with

$$
\hat{L}=-\partial_{x}+\left[\frac{3}{2} A^{2}-\frac{1}{2}-\frac{3 A^{2}}{2} \cosh ^{-2}(B x)\right], \quad r=-\Lambda^{2},
$$

whose eigenvalues turn out to be

$$
r_{n}=-\frac{1}{2}+B^{2}\left(L+2 L n-n^{2}\right),
$$

with $L(L+1)=3 A^{2} / 2 B^{2}$. Following the same lines as in the previous case, we conclude that if $A^{2}>1$ and $4 B^{2}<1, F_{I I}$ has three zeros that correspond to three equilibrium positions: one unstable equilibrium point at $x=0$ and two stable equilibria at $x= \pm d$, with

$$
d=\frac{1}{B} \operatorname{arccosh}\left[\sqrt{\frac{A^{2}-4 B^{2}}{A^{2}-1}}\right] .
$$

An interesting phenomenon occurs when $2 d$, the distance between the stable equilibrium points, is much less than the kink width. In this case, the translational mode is stable, which means that the kink does not feel the barrier created by the unstable equilibrium position.

When $A^{2}<1$ and $4 B^{2}>1$, we have a different situation: although $F(x)$ has three zeros, the equilibrium at $x=0$ is now stable and the two additional zeros would correspond to unstable equilibria, once again in the particle picture arising from the one-collective-variable calculation. However, as before, if the distance between zeros is much less than the kink width, the translational mode changes its stability and becomes unstable. This implies that the kink is not trapped by the potential well created by the $F_{I I}(x)$ at $x=0$.

\section{Case III}

Finally, let us analyze a third problem where we build a periodic perturbation by using Eq. (30) as a repeating structure, in the situation with three zeros $\left(A^{2}>1,4 B^{2}<1\right)$. The periodic forcing can be defined as $F_{I I I}=F_{I I}(x+2 n d)$, for $(2 n-1) d \leqslant x \leqslant(2 n+1) d$, with $n=0, \pm 1, \pm 2, \ldots$. When $d$ is much less than the kink width, based on the discussion in the preceding subsection, we can conclude that the kink will move in an almost flat potential. On the other hand, if we construct the periodic potential with the same amplitude but with a distance between zeros much larger than the kink width, the curvature of the effective potential will also be small.

\section{DISCUSSION AND CONCLUSIONS}

Having considered the three cases in the preceding section, we can benefit from the lessons learned from them and apply them to the problem we are dealing with: namely, the parametrical perturbation of Sec. II. As we showed there, the effective potential where the kink moves in the collective coordinate picture is almost flat for large and small values of the wavelength of the perturbation. On the other hand, the curvature of this potential has a maximum in the interval of parameters for which localization of the kink has been numerically observed $[11,14,24]$. In general, the kink moves in 
an effective periodic potential with wells and barriers. When the kink is inside a potential well, its width is reduced, whereas the opposite takes place when the kink is on a hill created by the perturbation. Once again, these changes of the kink size are larger in the interval of perturbation wavelengths where localization takes place.

Let us now compare the above results with the outcome of our second study about perturbations given by external inhomogeneous fields. If we have an external inhomogeneous perturbation that creates a potential well for the kink, the kink width is reduced as expected. On the other hand, we have shown that, if the external perturbation generates an unstable equilibrium position, the kink width is increased along with the number of activated internal modes. All these insights provide an accurate and complete picture of the length-scale competition phenomenon: The initial kinetic energy of the kink is transferred to the activated internal modes that generate large deformations of the kink shape, preventing the kink from propagating freely. The kink can also be trapped in a potential well, where even the translational mode cannot be activated; this phenomenon occurs simultaneously with the reduction of the kink width. Finally, we have shown that when the inhomogeneous external fields create several equilibrium positions, we can observe a phenomenon very reminiscent of length-scale competition. If the distance between the equilibrium positions is much less than the unperturbed kink width, then the kink does not feel the potential barriers and wells, and the kink moves in an almost flat potential; the same situation applies to the case when the distance between equilibrium positions is very large.

All the above considerations allow us to establish a scenario for the length-scale competition phenomenon by carrying over the analysis of the exactly solvable cases (Sec. III) to the parametric perturbation of Sec. II. This problem can not be treated directly with the operator approach because the shape of the kink is unknown in the presence of the perturbation. However, the overall behavior is basically the same: Indeed, both stable and unstable equilibria govern the dynamics because they are potential wells or hills, and lead to a reduction or enlargement of the kink width which in turn yields unstable internal modes and consequently unstabilize the kink solution. This must take place in the region where the stable width is different from the rest of the kink widthi.e., in the region $1.5 \leq k \leq 4$ (wavelengths $4.2 \geq \lambda \gtrsim 1.6$ ); cf. Fig. 1 and the values for $k_{m}$ and $l_{m}$ in Sec. II. This region corresponds to the largest curvatures of the effective potential (cf. Fig. 2) and therefore to the deepest potential wells, as was the case in the first problem studied in Sec. III. We are thus led to the conclusion that length-scale competition originates in the instability of the internal modes induced by the perturbation that become unstable in the parameter region mentioned above. This proposed scenario is in full agreement with the simulation results as well as with the numerical integration of the collective coordinate equations: Thus, Fig. 1 of [24] shows the stopping of the kink center coordinate $X(t)$ in the collective coordinate equations for $k=3$, whereas Fig. 2 of [24] shows that, in the numerical simulation of the full partial differential equation, there is a range of $k$ values in which the kink is trapped, due to the energy transfer from the kink motion to the internal modes excited by the potential.

Finally, a general conclusion of this work is the confirmation of the accuracy of the collective coordinate technique to describe nonlinear wave propagation under different types of perturbation. In this particular study we are presenting here, we have shown that, for the periodic case, this approach yields an effective dynamical system for the collective variables $X$ and $l$, whose equilibrium point structure is a clue to understanding the modification of the kink dynamics by the perturbation. We believe that this viewpoint of dynamical systems can be fruitful in many other problems where solitons play a role and can be accurately described by collective coordinates.

\section{ACKNOWLEDGMENTS}

This work was supported by MEC (Spain) under Grants MOSAICO and No. NAN2004-9087-C03-03 and by the Comunidad Autónoma de Madrid (Spain) under Grants No. UC3M-FI-05-007 and SIMUMAT-CM. The work by J.G. was done in part during a stay at Universidad Carlos III de Madrid, whose financial support is acknowledged, and S.C. is supported by the Consejería de Educación de la Comunidad Autónoma de Madrid and the Fondo Social Europeo.
[1] A. C. Scott, Nonlinear Science: Emergence and Dynamics of Coherent Structures, 2nd ed. (Oxford University Press, Oxford, 2003).

[2] N. J. Zabusky and M. D. Kruskal, Phys. Rev. Lett. 15, 240 (1965).

[3] J. K. Perring and T. R. H. Skyrme, Nucl. Phys. 31, 550 (1962).

[4] A. S. Davydov, Solitons in Molecular Systems, 2nd ed. (Springer-Verlag, Berlin, 1990).

[5] S. W. Englander, N. R. Kallenbach, A. J. Heeger, J. A. Krumhansl, and S. Litwin, Proc. Natl. Acad. Sci. U.S.A. 77, 7222 (1980)

[6] D. W. McLaughlin and A. C. Scott, Phys. Rev. A 18, 1652 (1978).
[7] A. D. Bruce and R. A. Cowley, Structural Phase Transitions (Taylor \& Francis, London, 1981).

[8] Encyclopedia of Nonlinear Science, edited by A. C. Scott (Routledge, New York, 2005), pp. 858-859.

[9] G. L. Lamb, Elements of Soliton Theory (Wiley-Interscience, New York, 1980).

[10] Yu. S. Kivshar and B. A. Malomed, Rev. Mod. Phys. 61, 763 (1989).

[11] A. Sánchez and A. R. Bishop, SIAM Rev. 40, 579 (1998).

[12] R. Scharf, Yu. S. Kivshar, A. Sánchez, and A. R. Bishop, Phys. Rev. A 45, R5369 (1992).

[13] A. Sánchez, R. Scharf, A. R. Bishop, and L. Vázquez, Phys. Rev. A 45, 6031 (1992). 
[14] A. Sánchez, A. R. Bishop, and F. Domínguez-Adame, Phys. Rev. E 49, 4603 (1994).

[15] R. Scharf and A. R. Bishop, Phys. Rev. E 47, 1375 (1993).

[16] D. Cai, A. R. Bishop, and A. Sánchez, Phys. Rev. E 48, 1447 (1993).

[17] J. A. González and B. D. Mello, Phys. Scr. 54, 14 (1996).

[18] J. A. González, B. D. Mello, L. I. Reyes, and L. E. Guerrero, Phys. Rev. Lett. 80, 1361 (1998).

[19] N. R. Quintero and A. Sánchez, Eur. Phys. J. B 6, 133 (1998).

[20] P. L. Christiansen, Yu. B. Gaididei, F. G. Mertens, and S. F. Mingaleev, Eur. Phys. J. B 19, 545 (2001).

[21] J. Garnier, Waves Random Media 11, 149 (2001).

[22] C. C. Hua and Y. Z. Liu, Commun. Theor. Phys. 37, 21 (2002)

[23] J. Garnier, Phys. Rev. B 68, 134302 (2003).
[24] S. Cuenda and A. Sánchez, Chaos 15, 023502 (2005).

[25] N. R. Quintero and E. Zamora-Sillero, Physica D 197, 63 (2004).

[26] J. A. González and J. A. Hołyst, Phys. Rev. B 45, 10338 (1992).

[27] A. R. Bishop, J. A. Krumhansl, and S. E. Trullinger, Physica D 1, 1 (1980).

[28] O. M. Braun and Yu. S. Kivshar, The Frenkel-Kontorova Model (Springer, Berlin, 2004).

[29] J. Goldstone and R. Jackiw, Phys. Rev. D 11, 1486 (1975).

[30] J. F. Currie, J. A. Krumhansl, A. R. Bishop, and S. E. Trullinger, Phys. Rev. B 22, 477 (1980).

[31] D. K. Campbell, J. F. Schonfeld, and C. A. Wingate, Physica D 9, 1 (1983). 